\title{
ENpHR: a Natronomonas halorhodopsin enhanced for optogenetic applications
}

\author{
Viviana Gradinaru ${ }^{1,2}$, Kimberly R. Thompson ${ }^{1}$ and Karl Deisseroth ${ }^{1,3, *}$ \\ ${ }^{1}$ Department of Bioengineering, Stanford University, W083 Clark Center, 318 Campus Drive West, \\ Stanford, CA, USA ( ${ }^{*}$ author for correspondence; e-mail: deissero@stanford.edu) \\ ${ }^{2}$ Program in Neuroscience, Stanford University, Stanford, CA, USA \\ ${ }^{3}$ Department of Psychiatry and Behavioral Sciences, Stanford University, Stanford, CA, USA
}

Received 22 May 2008; Revised 13 June 2008; Accepted 17 June 2008

Published online 2 August 2008

(C) The Author(s) 2008

\begin{abstract}
Temporally precise inhibition of distinct cell types in the intact nervous system has been enabled by the microbial halorhodopsin NpHR, a fast light-activated electrogenic $\mathrm{Cl}^{-}$pump. While neurons can be optically hyperpolarized and inhibited from firing action potentials at moderate NpHR expression levels, we have encountered challenges with pushing expression to extremely high levels, including apparent intracellular accumulations. We therefore sought to molecularly engineer NpHR to achieve strong expression without these cellular side effects. We found that high expression correlated with endoplasmic reticulum (ER) accumulation, and that under these conditions NpHR colocalized with ER proteins containing the KDEL ER retention sequence. We screened a number of different putative modulators of membrane trafficking and identified a combination of two motifs, an N-terminal signal peptide and a C-terminal ER export sequence, that markedly promoted membrane localization and ER export defined by confocal microscopy and whole-cell patch clamp. The modified NpHR displayed increased peak photocurrent in the absence of aggregations or toxicity, and potent optical inhibition was observed not only in vitro but also in vivo with thalamic single-unit recording. The new enhanced NpHR (eNpHR) allows safe, high-level expression in mammalian neurons, without toxicity and with augmented inhibitory function, in vitro and in vivo.
\end{abstract}

\section{Introduction}

A subset of naturally-occurring microbial opsin genes, originally characterized in non-neural systems, encode light-sensitive transmembrane ion conductance regulators (e.g., Hegemann et al., 1985; Kalaidzidis et al., 1998; Nagel et al., 2003; Zhang et al., 2008). If successfully adapted as a neuroscience technology, these proteins could be enormously significant, since controlling the membrane potential of targeted cell types with high temporal resolution may allow elucidation of cellular

Viviana Gradinaru and Kimberly R. Thompson contributed equally to this work. codes underlying neural circuit computation and behavior. Three functionally distinct classes of these microbial opsin genes have now been introduced to and adapted for neurobiology (VChR1, NpHR, and ChR2; discussed below). Among other important properties, all three operate on the millisecond timescale and can function in mammalian neurons without addition of exogenous chemical cofactors, since the chromophore for these proteins, all-trans retinal, appears to be already present at sufficient levels in mammalian brains (Zhang et al., 2006). Moreover, light and gene delivery challenges have been overcome, as integrated genetic, fiberoptic, and solid-state optical approaches have provided complementary technology to allow specific cell types, deep within the brain, to be controlled in freely 
behaving mammals (Adamantidis et al., 2007; Aravanis et al., 2007; Gradinaru et al., 2007).

First to be brought to neuroscience, the channelrhodopsin ChR2 allows blue light-induced action potentials to be triggered with millisecond-precision in neurons (Boyden et al., 2005), due to depolarizing cation flux through a light-gated pore (Nagel et al., 2003); this approach has since been shown to be versatile in many experimental systems ( $\mathrm{Li}$ et al., 2005; Nagel et al., 2005; Bi et al., 2006; Deisseroth et al., 2006; Ishizuka et al., 2006; Schroll et al., 2006; Zhang et al., 2006; Airan et al., 2007; Aravanis et al., 2007; Gradinaru et al., 2007; Hwang et al., 2007; Petreanu et al., 2007; Zhang and Oertner, 2007; Zhang et al., 2007a, b; Huber et al., 2008), including generation of transgenic mouse lines (Arenkiel et al., 2007; Wang et al., 2007) and probing neural codes underlying complex behavioral state transitions important in neuropsychiatric disease (Adamantidis et al., 2007). Second, we found that neurons targeted to express the lightactivated chloride pumping halorhodopsin from Natronomonas pharaonis (NpHR) can be hyperpolarized and inhibited from firing action potentials when exposed to yellow light (Zhang et al., 2007a); because of the excitation wavelength difference, ChR2 and NpHR can be coexpressed for bidirectional control and integrated with imaging and behavior (Gradinaru et al., 2007; Han and Boyden, 2007; Zhang et al., 2007a) even in intact tissue and behaving animals (Zhang et al., 2007a), and may turn out to be versatile across a range of in vitro and in vivo applications (reviewed in Gradinaru et al., 2007; Zhang et al., 2007b). Third, a yellow lightactivated channelrhodopsin gene was discovered and tested in mammalian neurons (VChR1; Zhang et al., 2008) that opens the door to combinatorial excitation experiments when used together with ChR2, described below. The properties of this third microbial tool also allow for deep penetration of redshifted excitation light, use of well-tolerated lowenergy photons for excitation, and improved integration with existing $\mathrm{Ca}^{2+}$ indicators.

Technical challenges still remain, including refining optical and cell type-specific targeting strategies, as well as tuning activation wavelengths and ion permeabilities for different classes of experiments. One major challenge in adapting tools across large evolutionary distances (e.g., to mammals from prokaryotes and simple eukaryotes such as Volvox carteri, Natronomonas pharaonis, and Chlamydomonas reinhardtii) is that expressing heterologous membrane proteins in mammalian cells can lead to poor folding, assembly, and trafficking. We have previously reported that at high expression levels, NpHR (codon optimized for mammalian expression) forms aggregates that could cause cellular toxicity (Gradinaru et al., 2007), and noted that this problem could be alleviated by returning to moderate expression levels, but this was not an ideal solution because large photocurrents are useful for efficient inhibition in a variety of experiments, especially for in vivo applications. Therefore, we report here on a strategy to increase the efficiency of NpHR membrane targeting to maximize photocurrents without aggregations or toxicity, even for high expression levels under strong promoters, in vitro and in vivo.

\section{Results}

In order to reduce the incidence of intracellular aggregates observed with NpHR at high expression levels, we first attempted to regulate distribution of the NpHR protein within the cell by using signal peptides from either ChR2 (Nagel et al., 2003) or the $\alpha$ and $\beta$ subunits of the nicotinic acetylcholine receptor (nAChR; Isenberg and Meyer, 1989; Bocquet et al., 2007), PDZ binding motifs (Zito et al., 1999; Weick et al., 2003; Guerrero et al., 2005), and actin binding motifs (Petrecca et al., 2000) (Fig. 1A). Although for most of the NpHR variants this strategy considerably reduced the number of cells with aggregates while maintaining photocurrents (Fig. 1B, C), aggregates were still observed and when present were as large as with wild-type NpHR. Next, after extensive analysis of the formation, distribution, and evolution of the NpHR aggregates in cultured neurons (data not shown) we observed that the localization of the aggregates appeared similar to ER localization in the soma and dendrites. Indeed, immunocytochemistry confirmed that NpHR aggregates colocalized with proteins containing the KDEL signal for ER retention (Fig. 2B, top row), while NpHR itself does not contain a KDEL motif or other known ER retention signals (e.g., the KKAA- or RSRR-class signals).

Transport along the secretory pathway, with ER export being the first step in the pathway, is crucial for surface expression of integral membrane proteins. Although some proteins can exit the ER 


\begin{tabular}{|c|c|}
\hline Construct & Molecular Modification \\
\hline NpHR & Codon optimized from bacterial NpHR \\
\hline nhNpHR & Non-humanized form: original codons from bacteria \\
\hline NpHR-ETQV & PDZ binding motif: ETQV (C-terminus) \\
\hline NpHR-PTPP & Actin-binding protein filamin motif: PTPP (C-terminus) \\
\hline $\mathrm{SP}_{\text {BnAChR }}-\mathrm{NpHR}$ & $\begin{array}{l}\text { Signal peptide from nicotinic acetylcholine receptor ( } \beta \text { subunit): } \\
\text { MRGTPLLLWVSLFSLLQD ( N-terminus) }\end{array}$ \\
\hline $\mathrm{NpHR}-\mathrm{ER}_{1}$ & ER export signal: VLGSL (C-terminus) \\
\hline $\mathrm{SP}_{\text {BnaChR-NpHR-ER, }}$ & $\begin{array}{l}\text { Signal peptide from nicotinic acetylcholine receptor ( } \beta \text { subunit): } \\
\text { MRGTPLLLVVLFSLLQD ( N-terminus) } \\
\text { ER export signal: VLGSL (C-terminus) }\end{array}$ \\
\hline $\mathrm{SP}_{\text {BnAChR }}-\mathrm{NpHR}-\mathrm{ER}_{2}$ & $\begin{array}{l}\text { Signal peptide from nicotinic acetylcholine receptor ( } \beta \text { subunit): } \\
\text { MRGTPLLLWVLFSLLQD ( N-terminus) } \\
\text { ER export signal: FCYENEV ( C-terminus) }\end{array}$ \\
\hline $\mathrm{SP}_{\text {BnAChR }}-\mathrm{NpHR}-\mathrm{ER}_{3}$ & $\begin{array}{l}\text { Signal peptide from nicotinic acetylcholine receptor ( } \beta \text { subunit): } \\
\text { MRGTPLLLWVSLFSLLQD ( N-terminus) } \\
\text { ER export signal: RLQVMIQEAYI ( C-terminus) }\end{array}$ \\
\hline $\mathrm{SP}_{\mathrm{ChR}_{2}-\mathrm{NpHR}}$ & Signal peptide from ChR2: DYGGALSAVGRELL ( $\mathrm{N}$-terminus) \\
\hline $\mathrm{SP}_{\text {anAChR }}-\mathrm{NpHR}$ & $\begin{array}{l}\text { Signal peptide from nicotinic acetylcholine receptor (a subunit): } \\
\text { MGLRALMLWLLAAAGLVRESLQG ( N-terminus) }\end{array}$ \\
\hline NpHR-VSNL & PDZ binding motif: VSNL (C-terminus) \\
\hline
\end{tabular}

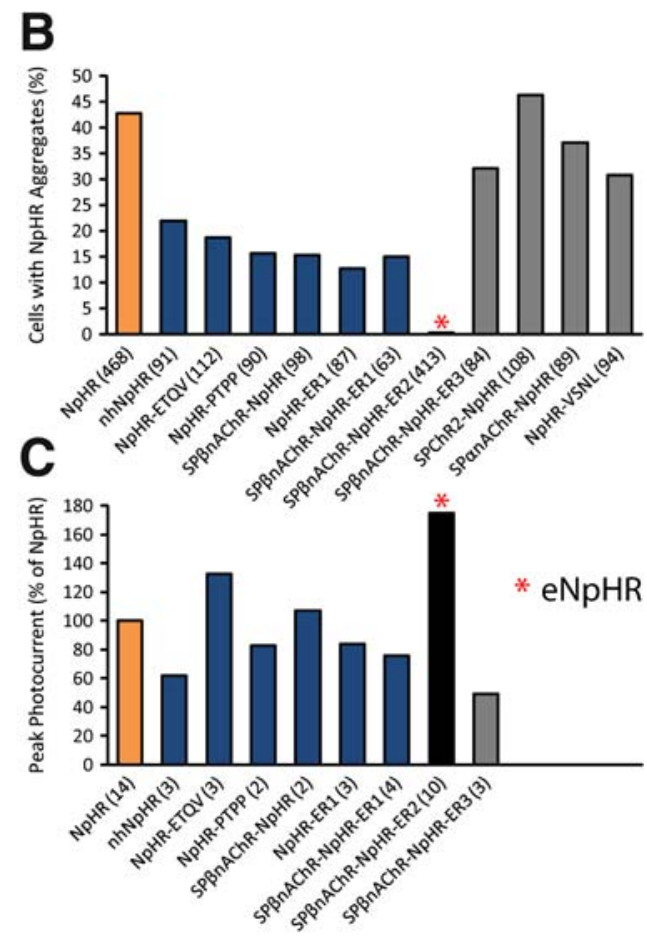

Fig. 1. Protein aggregation and photocurrents altered by changes in intracellular targeting of NpHR. (A) Table of screened intracellular targeting strategies using $\mathrm{N}$ - and $\mathrm{C}$-terminal peptides fused to NpHR. (B) The fraction of neurons containing one or more NpHR aggregates was determined in cultured hippocampal neurons transduced with quantitatively titer-matched viral CaMKII $\alpha:$ NpHR-EYFP constructs, and allowed to express for 10 days. Compared to the unmodified NpHR (orange), some constructs (gray) had little effect on aggregates while others (blue) partially reduced aggregate incidence. Notably, for one construct $\left(^{*}\right)$ aggregates and toxicity were virtually abolished (only a single possible aggregate observed in $>400$ neurons). Data shown are relative to wild-type NpHR, and the number of neurons sampled for each construct is shown in parenthesis after the construct label. (C) Functionality was assessed by whole-cell patch clamp (for details see "Methods" and Fig. 3B). All the constructs were functional as indicated by photocurrents that were comparable to the original NpHR, but one construct, the optimal construct from $(B)\left({ }^{*}\right)$, gave rise to significantly higher photocurrent per cell than all other variants (eNpHR). Data shown are relative to wild-type NpHR, and the number of neurons sampled for each construct is shown in parenthesis after the construct label.

by bulk flow, ER export of membrane proteins can be impaired if the protein is either misfolded or lacks specific export signals (Li et al., 2000; Ellgaard and Helenius, 2003). Because NpHR is functional in mammalian neurons even in vivo (Zhang et al., 2007a) we hypothesized that aggregate formation might be due to lack of an ER export signal rather than frank misfolding, and therefore sought to determine if adding different ER export signals to the NpHR sequence would abolish aggregate formation. C-terminal ER export signals have been shown to be important for efficient processing and surface expression of many membrane proteins (Farhan et al., 2008). Additionally, previous work has found that when the C-terminal ER export signals on Kv1.4 (VXXSL) or Kir2.1 (FCYENEV) are either mutated or deleted, the resulting protein forms large spheroidal intracellular accumulations similar to NpHR aggregates, and corresponding channel activity is reduced due to lower protein levels in the plasma membrane (Levitan and Takimoto, 2000; Ma et al., 2001; Stockklausner et al., 2001). Moreover, suggesting that functionality can be transferred solely with these motifs, the FCYENEV sequence accelerated surface expression and increased current levels for the lobster shal potassium channel (Kv4) when added to the C-terminus (Zhang and Harris-Warrick, 2004). Indeed, in the course of our modification screen we found that adding FCYENEV to the $\mathrm{NpHR}$ C-terminus along with the signal peptide from the $\beta$ subunit of the $\mathrm{nAChR}$ to the $\mathrm{NpHR}$ $\mathrm{N}$-terminus prevented aggregate formation (Fig. 1B, asterisk), with such markedly improved 
A

Signal peptide for membrane insertion:

ER export for preventing aggregates: ..MRGTPLLLVVSLFSLLQD.. (from nAChR) ..FCYENEV.. (from Kir2.1)

\section{Promoter MRGTPLLLVVSLFSLLQD NpHR EYFP FCYENEV}

B
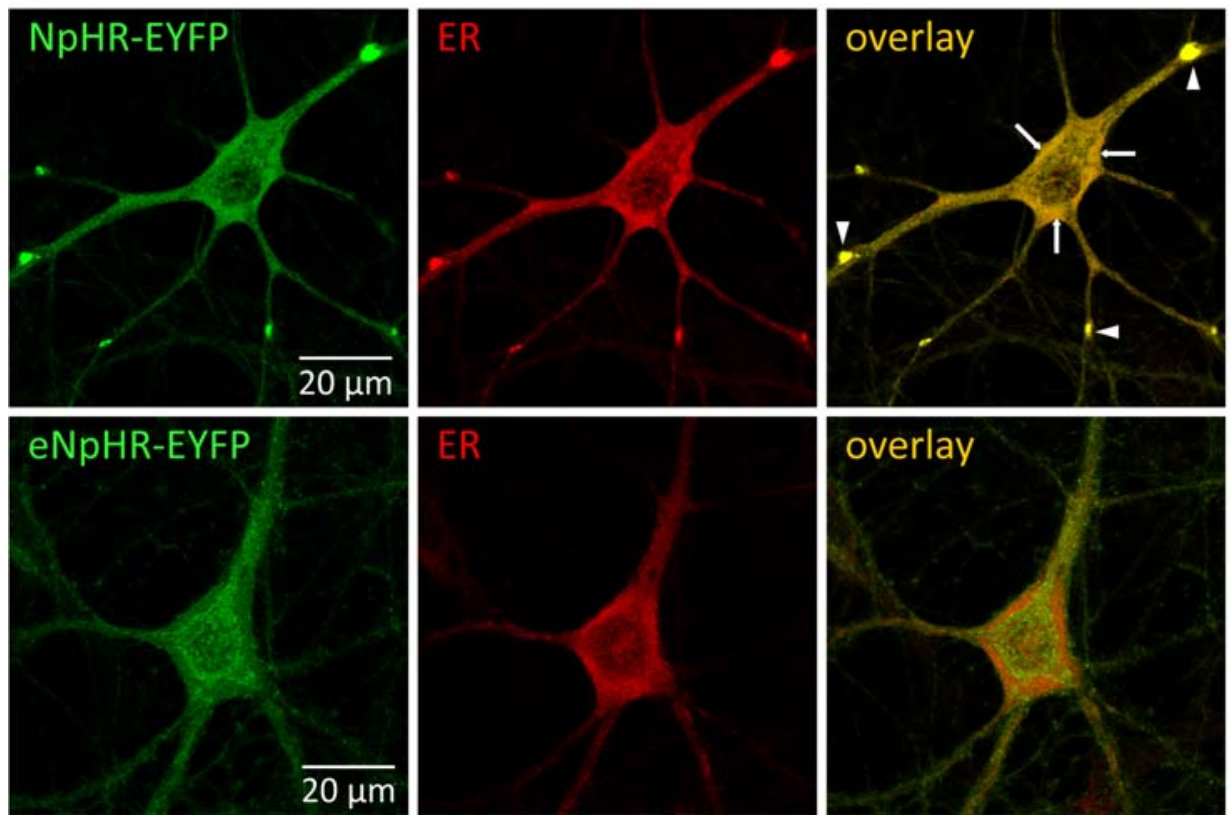
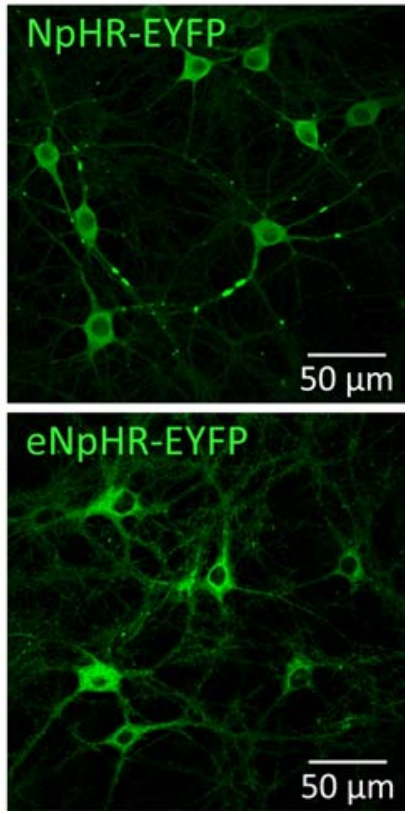

Fig. 2. Intracellular targeting of eNpHR. (A) Primary structure of the selected construct (eNpHR) showing addition of the N-terminal signal peptide derived from $n A C h R$ and the C-terminal ER export signal derived from Kir2.1. Expression here was driven by the CaMKIl $\alpha$ promoter and visualized by fusion to EYFP. (B) Top row: Untargeted NpHR (green) colocalized with somatic ER (KDEL ER protein staining in red; overlap indicated in yellow and by arrows) and also notably aggregated in ER-rich regions of the dendrites (overlap indicated in yellow and by arrowheads). Bottom row: Little colocalization of eNpHR with somatic ER staining could be found, and indeed pronounced accumulations of ER staining in eNpHR dendrites were not observed. Right column: representative images of neuronal populations expressing NpHR and eNpHR. The neurons were infected with quantitatively titer-matched CaMKIl $\alpha$-NpHR-EYFP or CaMKIl $\alpha$-eNpHR-EYFP.

properties that we named the resulting tool eNpHR (enhanced NpHR; Fig. 2A) and studied its behavior further (summarized in Figs. 2-4).

First, we found that in contrast to NpHR, eNpHR did not colocalize with ER proteins (Fig. 2B), or show evidence of toxicity even at high expression levels. eNpHR appeared to be present in somatic Golgi structures, typical for transmembrane proteins as they are packaged for transport (Ma et al., 2001; Horton and Ehlers, 2003; Hofherr et al., 2005). Notably, eNpHR showed more surface membrane localization as defined by whole-cell photocurrents (Fig. 3). Indeed, presumably due to increased export from the ER and increased membrane localization, eNpHR displayed significantly higher photocurrents compared to NpHR in cultured neurons infected with quantitatively titer-matched virus levels and allowed to express for the same amount of time (Fig. 3: NpHR: $38.9 \pm 6.8 \mathrm{pA}$; eNpHR $68.1 \pm 7.2 \mathrm{pA}$; mean \pm SEM; unpaired $t$-test $P=0.008$ ), while membrane resistance was indistinguishable in the two groups (NpHR: $113.5 \pm$ $13.9 \mathrm{~m} \Omega$; eNpHR: $116.8 \pm 13.9 \mathrm{~m} \Omega$; unpaired $t$-test $P=0.87$ ).

To assess eNpHR function in the setting of high in vivo expression levels, we injected highly 

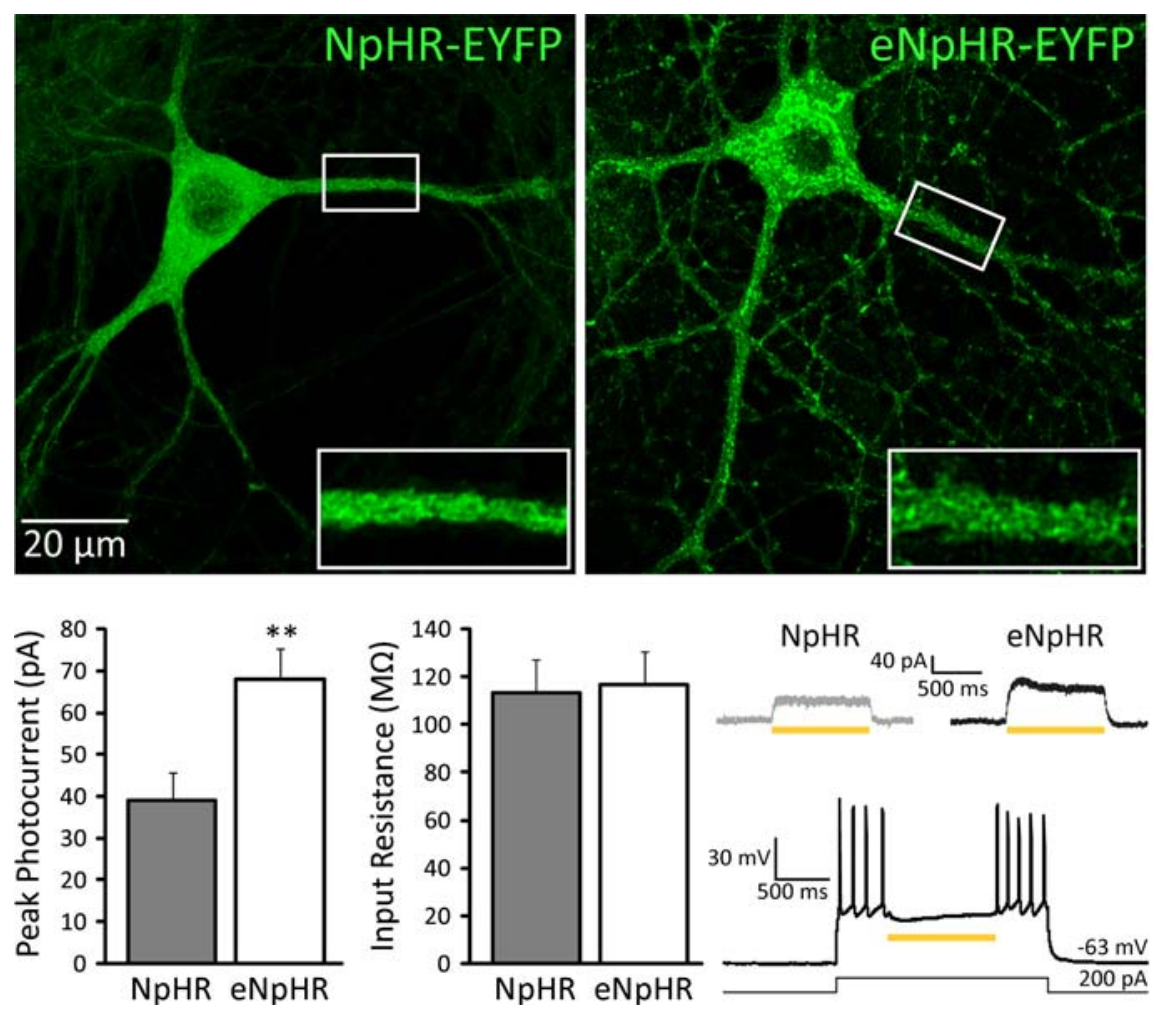

Fig. 3. Summary of eNpHR functional properties. Summary of electrophysiological properties of NpHR and eNpHR in cultured hippocampal neurons. Top: Representative confocal images in cultured hippocampal neurons revealed that like a typical membrane protein, eNpHR did not appear to fill cytoplasm like NpHR (left) (right). NpHR and eNpHR were expressed for 10 days in cultured hippocampal neurons. Insets: magnified views of selected regions. Bottom: $593 \mathrm{~nm}$ light (yellow bar) induced outward photocurrents (top right: sample traces in voltage clamp), with eNpHR evoking significantly stronger photocurrents per cell than NpHR (left bar graph; NpHR: $38.9 \pm 6.8$ pA; eNpHR: $68.1 \pm$ $7.2 \mathrm{pA}$; unpaired $t$-test $P=0.008$ ). Viral titers were quantitatively matched across groups (see "Methods"). Membrane input resistance was similar for all neurons patched (right bar graph; NpHR: $113.5 \pm 13.9 \mathrm{~m} \Omega$; eNpHR: $116.8 \pm 13.9 \mathrm{~m} \Omega$; unpaired $t$-test $P=0.87$ ). Values plotted are mean \pm SEM; $n=12$ for NpHR, $n=10$ for eNpHR. Bottom right: Illumination with yellow light as expected sufficed to inhibit spiking induced by current injection in eNpHR+ neurons.

concentrated and quantitatively titer-matched virus for both $\mathrm{NpHR}$ and eNpHR under the strong CaMKIl $\alpha$ promoter into the CA1 region of adult mouse hippocampus. While NpHR showed aggregates after 10 days of expression in vivo, eNpHR did not show any aggregates or affect cellular integrity, confirming the tolerability and improved targeting of the engineered protein even at these very high expression levels (Fig. 4A, top row); enhanced membrane localization of eNpHR in vivo was also evident in dendrites (Fig. 4A, bottom row). To confirm functionality, we conducted in vivo recordings using a combined optical fiber/electrode "optrode" previously described (Gradinaru et al., 2007), and found that activity of single units in adult mouse thalamus expressing eNpHR (Fig. 4B, confocal image) could be readily and reversibly inhibited with yellow light (Fig. 4B, middle) in vivo; no such effect was seen in non-transduced tissue (Fig. 4B, bottom).

\section{Discussion}

In this study we have identified and corrected a major limiting factor in the application of optogenetic inhibition (complementary results have been obtained with our colleagues at Duke University; Zhao et al., 2008). We traced the problem associated with high NpHR expression back to a membrane trafficking complication (Figs. 1 and 2), 
A
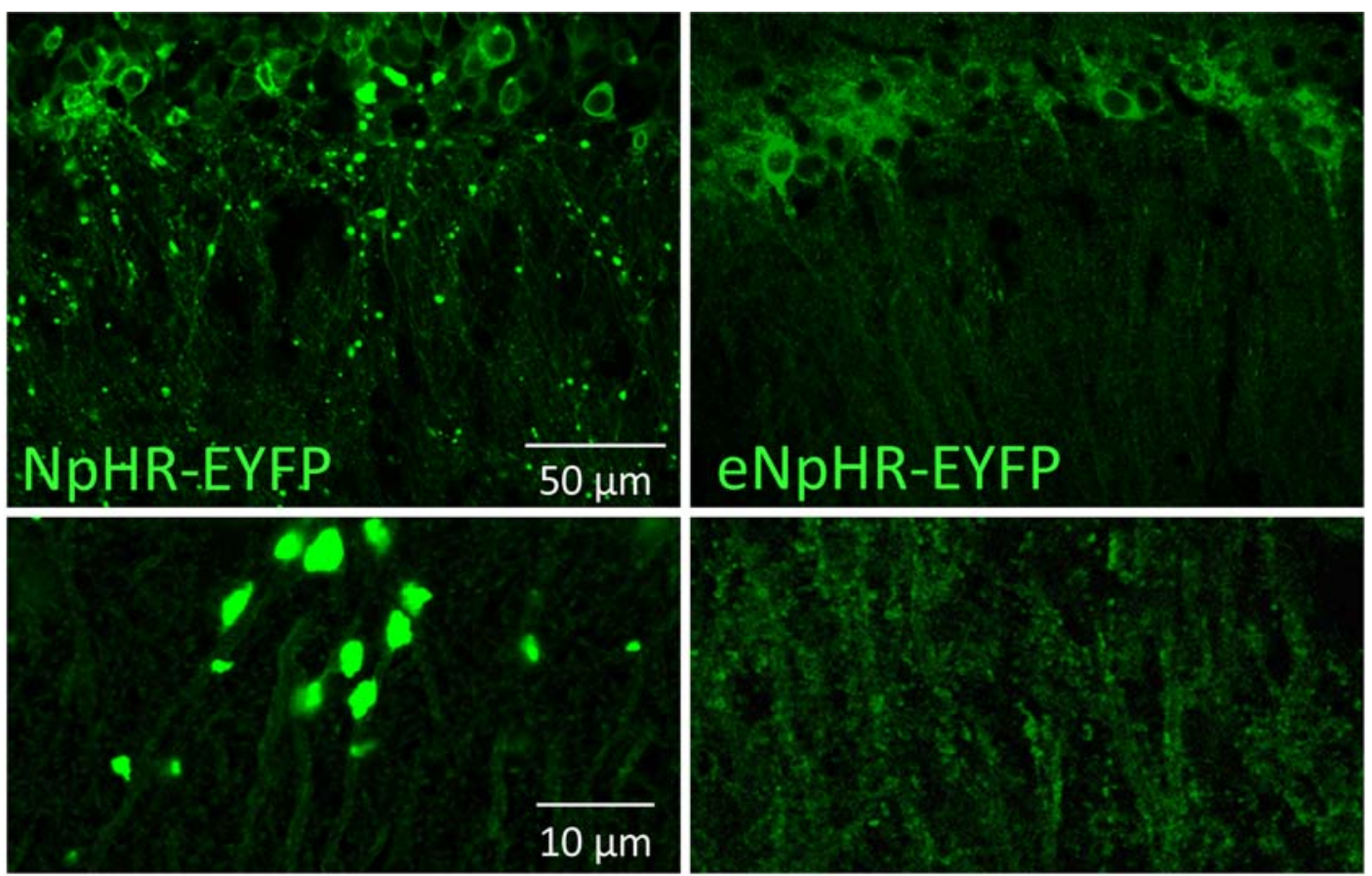

B

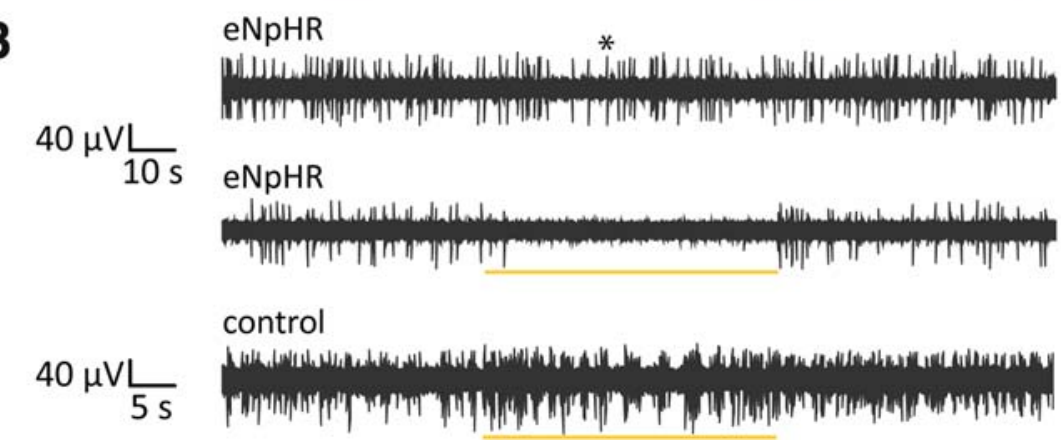

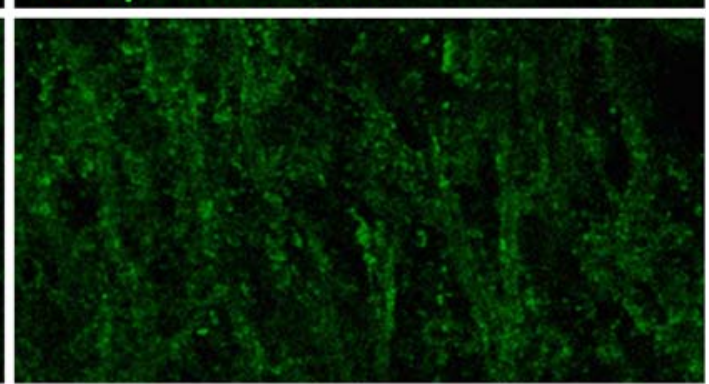
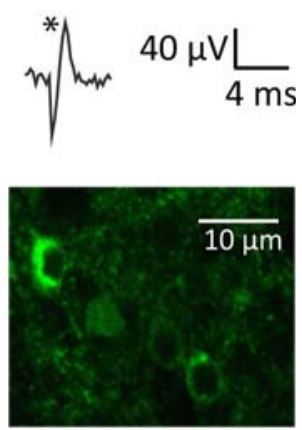

Fig. 4. In vivo function of eNpHR at high expression level. (A) Confocal images showing NpHR and eNpHR expression in rodent hippocampal CA1. NpHR aggregations were clearly visualized after 10 days of strong expression of high titer virus (left) while eNpHR showed no signs of aggregates or toxicity (right) with quantitatively matched viral titers (see "Methods"). Lower panels: magnified views of the dendritic layer. Compared to NpHR, eNpHR revealed not only absence of aggregates but also more membrane localization in distal processes in vivo. (B) Simultaneous optical stimulation and electrophysiology in living mice demonstrates eNpHR potency in vivo: single unit recordings in deep brain structures. Yellow illumination delivered by the optrode method (Gradinaru et al., 2007) in vivo inhibited electrical activity in thalamus previously transduced with eNpHR by lentiviral stereotactic injection (middle trace). Top trace: same thalamic region, recording without illumination. Bottom trace: control recording $1 \mathrm{~mm}$ ventral and anterior from the eNpHR injection site. As expected, in nontransduced tissue, light did not inhibit recorded spikes. Confocal image: eNpHR expression in the thalamus (same animal). Inset: expanded view of a spike from unit $\left(^{*}\right)$ represented in the top trace.

tested a large number of possible solutions (Fig. 1), and validated the efficacy of the best strategy both in vitro (Figs. 1-3) and in vivo (Fig. 4). We found that eNpHR completely abolished accumulations seen at very high expression levels with the original $\mathrm{NpHR}$, apparently in part by allowing normal export of NpHR from the ER (assessed by confocal imaging) and by driving increased surface membrane expression (validated by quantified photocurrents). At this point we recommend use of eNpHR for all applications, and certainly those involving high expression levels in mammalian neurons, including 
transgenic mouse line generation (Zhao et al., 2008) and viral transduction approaches. We also anticipate that these modifications may enhance the expression of other microbial opsins at high levels and over long durations, pointing to the likely utility of generating similarly enhanced versions of ChR2 and VChR1.

The altered properties of eNpHR as described here clearly do not simply represent a subtle quantitative change in performance, but rather a distinct step in the development of this optogenetic technology. Future improvements could incrementally further advance eNpHR function, perhaps including the Golgi export signals from Kir2.1 (Stockklausner and Klocker, 2003; Hofherr et al., 2005), subcellular localization motifs (as in Gradinaru et al., 2007), and mutations that shift wavelength dependence, kinetics, light sensitivity, and ion selectivity. For example, blueshifting ChR2 and redshifting eNpHR and VChR1 will improve the ease with which combinatorial experiments are conducted, and a roadmap for the key residues likely to be involved and the type of changes likely to be helpful in this regard has been described (Zhang et al., 2008).

Finally, it has been noted (Zhang et al., 2008) that VChR1 and ChR2 (representing yellow light excitation and blue light excitation, respectively) when used together will allow combinatorial tests of the importance of specific activity patterns in interacting cell types. For example, a principal cell population can be recruited with VChR1/yellow light, in the presence or absence of precisely patterned activity in a candidate modulatory cell type driven by added blue light/ChR2. Combinatorial experiments are important to consider now for eNpHR as well, given its improved functionality in vitro and in vivo. For example, coexpression of eNpHR and ChR2 in the same cell type could allow testing the necessity and sufficiency of that specific cell type in neural circuit or animal behavior. Moreover, epistatic relationships among different cell groups within a neuronal network could be probed by expressing eNpHR and $\mathrm{ChR} 2$ in different cell types and determining if the functional significance of cell population A excitation is expressed through or "read out" via a candidate downstream cell population $\mathrm{B}$; this hypothesis could be tested by reversibly inhibiting cell population $B$ (yellow light/eNpHR) in the presence of population $A$ activation (blue light/ChR2). In this way the causal neural codes underlying circuit computation and behavior may be slowly assembled, moving toward the long term goal of understanding how neural system properties emerge from component dynamics, both in health and disease.

\section{Methods}

\section{DNA constructs}

All NpHR variants were produced by PCR amplification of the NpHR-EYFP construct previously published (Zhang et al., 2007a) and cloned in-frame into the Agel and EcoRI restriction sites of a lentivirus carrying the CaMKII $\alpha$ promoter according to standard molecular biology protocols. All constructs were fully sequenced to check for accuracy of the cloning procedure. The map for eNpHR is available online at www.optogenetics.org.

\section{Lentivirus preparation and titering}

Lentiviruses for cultured neuron infection and for in vivo injection were produced as previously described (Zhang et al., 2007a). Viral titering was performed in HEK293 cells that were grown in 24-well plates and inoculated with 5-fold serial dilutions in the presence of polybrene $(8 \mu \mathrm{g} / \mu \mathrm{l})$. After 4 days, cultures were resuspended in PBS and sorted for EYFP fluorescence on a FACScan flow cytometer (collecting 20,000 events per sample) followed by analysis using FlowJo software (Ashland, OR). The titer of the virus was determined as follows: [ $\%$ of infected cells $) \times$ (total number of cells in well) $\times$ (dilution factor)]/(volume of inoculum added to cells) $=$ infectious units $/ \mathrm{ml}$. The titer of viruses for culture infection was $10^{5} \mathrm{i}$ i.u./ml. The titer of concentrated virus for in vivo injection was $10^{10}$ i.u./ml.

\section{Hippocampal cultures}

Primary cultured hippocampal neurons were prepared from P0 Spague-Dawley rat pups. The CA1 and CA3 regions were isolated, digested with 0.4 $\mathrm{mg} / \mathrm{ml}$ papain (Worthington, Lakewood, $\mathrm{NJ}$ ), and plated onto glass coverslips precoated with 1:30 Matrigel (Beckton Dickinson Labware, Bedford, MA) at a density of $65,000 / \mathrm{cm}^{2}$. Cultures were maintained in a $5 \% \mathrm{CO}_{2}$ humid incubator with Neurobasal-A media (Invitrogen Carlsbad, CA) containing 1.25\% FBS (Hyclone, Logan, UT), 4\% B-27 supplement (Gibco, Grand Island, NY), 2 mM 
Glutamax (Gibco), and FUDR (2 mg/ml, Sigma, St. Louis, MO).

\section{In vitro electrophysiology}

Hippocampal cultures grown on coverslips were transduced at 4 div with titer-matched viruses for all CaMKII $\alpha$-NpHR-EYFP constructs (final dilution $10^{4}$ i.u./ml in neuronal growth media) and allowed to express for 10 days. Whole-cell patch clamp recordings were performed as previously described (Gradinaru et al., 2007) (intracellular solution: 129 $\mathrm{mM}$ K-gluconate, $10 \mathrm{mM}$ HEPES, $10 \mathrm{mM} \mathrm{KCl}, 4$ mM MgATP, $0.3 \mathrm{mM} \mathrm{Na}_{3} \mathrm{GTP}$, titrated to $\mathrm{pH}$ 7.2; extracellular solution, tyrode: $125 \mathrm{mM} \mathrm{NaCl}, 2 \mathrm{mM}$ $\mathrm{KCl}, 3 \mathrm{mM} \mathrm{CaCl}_{2}, 1 \mathrm{mM} \mathrm{MgCl}_{2}, 30 \mathrm{mM}$ glucose, and $25 \mathrm{mM}$ HEPES, titrated to $\mathrm{pH}$ 7.3). Light $\left(7 \mathrm{~mW} / \mathrm{mm}^{2}\right)$ was delivered from a $300 \mathrm{~W}$ DG-4 lamp (Sutter Instruments, Novato, CA) through a $593 \mathrm{~nm} \pm 20 \mathrm{~nm}$ filter (Semrock, Rochester, NY) and a 20X/0.45NA air objective (Olympus, Center Valley, PA).

\section{Immunostaining and aggregate count}

Primary hippocampal cultures grown on coverslips were infected at 4 div with titer matched virus (final dilution $10^{4}$ i.u./ml in neuronal growth media). At 14 div cultures were fixed for $30 \mathrm{~min}$ with ice-cold $4 \%$ paraformaldehyde and then permeabilized for 30 min with $0.4 \%$ saponin in $2 \%$ normal donkey serum (NDS). Primary antibody incubations were performed overnight at $4^{\circ} \mathrm{C}$ using a monoclonal marker of endoplasmic reticulum recognizing endogenous ER-resident proteins containing the KDEL retention signal (KDEL 1:200, Abcam, Cambridge, MA). For detection we used Cy3-conjugated secondary antibodies (Jackson Laboratories, West Grove, PA) in 2\% NDS for $1 \mathrm{~h}$ at room temperature. Close-up images of neurons were taken on a Leica confocal microscope using a 63X/ 1.4NA oil objective. The percentage of cells with aggregates was estimated by an unbiased count over multiple fields and coverslips.

\section{Stereotactic injection into the rodent brain}

Adult C57BL/6 mice were housed according to the Laboratory Vertebrate Animal protocols at Stanford. All surgeries were performed under aseptic conditions. The animals were anesthetized with intraperitoneal injections of ketamine $(80 \mathrm{mg} / \mathrm{kg}) /$ xylazine (15-20 mg/kg) cocktail (Sigma). The head was shaved, cleaned with $70 \%$ ethanol and betadine and then placed in a stereotactic apparatus (Kopf Instruments, Tujunga, CA; Olympus stereomicroscope). Ophthalmic ointment was applied to prevent eye drying. A midline scalp incision was made and then a small craniotomy was performed using a drill mounted on the stereotactic apparatus (Fine Science Tools, Foster City, CA). The virus was delivered using a $10 \mu \mathrm{l}$ syringe and a thin 34 gauge metal needle; the injection volume and flow rate $(1 \mu \mathrm{l}$ at $0.1 \mu \mathrm{l} / \mathrm{min})$ was controlled with an injection pump from World Precision Instruments (Sarasota, FL). After injection the needle was left in place for 10 additional minutes and then slowly withdrawn. The skin was glued back with Vetbond tissue adhesive. The animal was kept on a heating pad until it recovered from anesthetic. Buprenorphine $(0.03 \mathrm{mg} / \mathrm{kg})$ was given subcutaneously following the surgical procedure to minimize discomfort. For hippocampal slice imaging: $1 \mu \mathrm{l}$ of concentrated lentivirus $\left(10^{10}\right.$ i.u./ml) carrying NpHR or eNpHR under the CaMKII $\alpha$ promoter was microinjected into the CA1 region of the left and right adult mouse hippocampus, respectively (anteroposterior, $-2.0 \mathrm{~mm}$ from bregma; lateral, $\pm 1.5 \mathrm{~mm}$; ventral, 2 $\mathrm{mm}$ ). For in vivo electrophysiology: $1 \mu \mathrm{l}$ of eNpHR $\left(10^{10} \mathrm{i} . u\right.$. $\left./ \mathrm{ml}\right)$ virus was injected in the adult mouse thalamus (anteroposterior $-1.8, \mathrm{~mm}$ from bregma; lateral, $1.5 \mathrm{~mm}$; ventral, $3.5 \mathrm{~mm}$ ).

\section{Slice preparation and confocal imaging}

For preparation of brain slices, mice were sacrificed 10 days after viral injection. Acute coronal brain slices $(250 \mu \mathrm{m})$ were prepared in ice-cold cutting solution $\left(64 \mathrm{mM} \mathrm{NaCl}, 25 \mathrm{mM} \mathrm{NaHCO}_{3}, 10\right.$ $\mathrm{mM}$ glucose, $120 \mathrm{mM}$ sucrose, $2.5 \mathrm{mM} \mathrm{KCl}, 1.25$ $\mathrm{mM} \mathrm{NaH}{ }_{2} \mathrm{PO}_{4}, 0.5 \mathrm{mM} \mathrm{CaCl}_{2}, 7 \mathrm{mM} \mathrm{MgCl}_{2}$, and equilibrated with $95 \% \mathrm{O}_{2} / 5 \% \mathrm{CO}_{2}$ ) using a vibratome (VT1000S, Leica). The slices were then fixed for $1 \mathrm{~h}$ in $4 \%$ paraformaldehyde, washed with PBS, and mounted on microscope slides. Single confocal optical sections through the CA1 region or thalamus were acquired using a 40X/1.4NA oil objective on a Leica confocal microscope.

\section{In vivo multiunit recordings}

Simultaneous optical stimulation and electrical recording in living mice was done as described previously (Gradinaru et al., 2007) using an optrode composed of an extracellular tungsten electrode (1 M $\Omega, \sim 125 \mu \mathrm{m}$ ) tightly attached to an optical fiber $(\sim 200 \mu \mathrm{m}$, ThorLabs, Newton, NJ) with the tip of the electrode deeper $(\sim 0.3 \mathrm{~mm})$ than the tip of the fiber, 
to ensure illumination of the recorded neurons. The fiberoptic was coupled to a $561 \mathrm{~nm}$ laser diode from CrystaLaser (Reno, NV). Single unit recordings were done in animals anesthetized with intraperitoneal injections of ketamine $(80 \mathrm{mg} / \mathrm{kg}) / x y$ lazine $(15-20$ $\mathrm{mg} / \mathrm{kg}$ ) cocktail (Sigma). pClamp 10 and a Digidata 1322A board (Axon Instruments, Sunnyvale, CA) were used to both collect data and generate light pulses through the fiber. The recorded signal was band pass filtered at $300 \mathrm{~Hz}$ low $/ 5 \mathrm{kHz}$ high (1800 Microelectrode AC Amplifier, A-M Systems). For precise placement of the fiber/electrode pair, stereotactic instrumentation (Kopf; Olympus stereomicroscope) was used. Immediately after recordings the animal was sacrificed and brain slices were prepared as described above to check for opsin expression and accurate placement of the optrode.

\section{Acknowledgments}

K.D. is supported by CIRM, McKnight, Coulter, Klingenstein, NSF, NIMH, NIDA, the NIH Pioneer Award, and the Kinetics Foundation. V.G. is supported by a Stanford Graduate Fellowship. K.R.T. is supported by NARSAD. We thank Feng Zhang and Joanna Mattis for providing us with the nhNpHR construct, and Andrew Hsu for assistance with cloning. We also thank the entire Deisseroth lab for useful discussions. The materials and methods described herein are freely distributed and supported by the authors (www.stanford.edu/group/dlab).

\section{Open Access}

This article is distributed under the terms of the Creative Commons Attribution Noncommercial License which permits any noncommercial use, distribution, and reproduction in any medium, provided the original author(s) and source are credited.

\section{References}

Adamantidis, A. R., Zhang, F., Aravanis, A. M., Deisseroth, K., and de Lecea, L. (2007). Neural substrates of awakening probed with optogenetic control of hypocretin neurons. Nature $450,420-424$.
Airan, R. D., Hu, E. S., Vijaykumar, R., Roy, M., Meltzer, L. A., and Deisseroth, K. (2007). Integration of light-controlled neuronal firing and fast circuit imaging. Curr. Opin. Neurobiol. 17, 587-592.

Aravanis, A. M., Wang, L. P., Zhang, F., Meltzer, L. A., Mogri, M. Z., Schneider, M. B., and Deisseroth, K. (2007). An optical neural interface: In vivo control of rodent motor cortex with integrated fiberoptic and optogenetic technology. J. Neural. Eng. 4, S143-S156.

Arenkiel, B. R., Peca, J., Davison, I. G., Feliciano, C., Deisseroth, K., Augustine, G. J., Ehlers, M. D., and Feng, G. (2007). In vivo light-induced activation of neural circuitry in transgenic mice expressing channelrhodopsin-2. Neuron 54, 205-218.

Bi, A., Cui, J., Ma, Y. P., Olshevskaya, E., Pu, M., Dizhoor, A. M., and Pan, Z. H. (2006). Ectopic expression of a microbial-type rhodopsin restores visual responses in mice with photoreceptor degeneration. Neuron 50, 23-33.

Bocquet, N., Prado de Carvalho, L., Cartaud, J., Neyton, J., Le Poupon, C., Taly, A., Grutter, T., Changeux, J. P., and Corringer, P. J. (2007). A prokaryotic proton-gated ion channel from the nicotinic acetylcholine receptor family. Nature 445, 116-119.

Boyden, E. S., Zhang, F., Bamberg, E., Nagel, G., and Deisseroth, K. (2005). Millisecondtimescale, genetically targeted optical control of neural activity. Nat. Neurosci. 8, 1263-1268.

Deisseroth, K., Feng, G., Majewska, A. K., Miesenbock, G., Ting, A., and Schnitzer, M. J. (2006). Next-generation optical technologies for illuminating genetically targeted brain circuits. J. Neurosci. 26, 10380-10386.

Ellgaard, L. and Helenius, A. (2003). Quality control in the endoplasmic reticulum. Nat. Rev. Mol. Cell. Biol. 4, 181-191.

Farhan, H., Reiterer, V., Kriz, A., Hauri, H. P., Pavelka, M., Sitte, H. H., and Freissmuth, M. (2008). Signal-dependent export of GABA transporter 1 from the ER-Golgi intermediate compartment is specified by a C-terminal motif. J. Cell Sci. 121, 753-761.

Gradinaru, V., Thompson, K. R., Zhang, F., Mogri, M., Kay, K., Schneider, M. B., and Deisseroth, K. (2007). Targeting and readout strategies for fast optical neural control in vitro and in vivo. J. Neurosci. 27, 14231-14238. 
Guerrero, G., Reiff, D. F., Agarwal, G., Ball, R. W., Borst, A., Goodman, C. S., and Isacoff, E. Y. (2005). Heterogeneity in synaptic transmission along a Drosophila larval motor axon. Nat. Neurosci. 8, 1188-1196.

Han, X. and Boyden, E. S. (2007). Multiple-color optical activation, silencing, and desynchronization of neural activity, with single-spike temporal resolution. PLoS ONE 2, e299.

Hegemann, P., Oesterhelt, D., and Steiner, M. (1985). The photocycle of the chloride pump halorhodopsin. I. Azidecatalyzed deprotonation of the chromophore is a side reaction of photocycle intermediates inactivating the pump. EMBO J. 4z, 2347-2350.

Hofherr, A., Fakler, B., and Klocker, N. (2005). Selective Golgi export of Kir2.1 controls the stoichiometry of functional Kir2.x channel heteromers. J. Cell Sci. 118, 1935-1943.

Horton, A. C. and Ehlers, M. D. (2003). Dual modes of endoplasmic reticulum-to-Golgi transport in dendrites revealed by live-cell imaging. J. Neurosci. 23, 6188-6199.

Huber, D., Petreanu, L., Ghitani, N., Ranade, S., Hromadka, T., Mainen, Z., and Svoboda, K. (2008). Sparse optical microstimulation in barrel cortex drives learned behaviour in freely moving mice. Nature 451, 61-64.

Hwang, R. Y., Zhong, L., Xu, Y., Johnson, T., Zhang, F., Deisseroth, K., and Tracey, W. D. (2007). Nociceptive neurons protect Drosophila larvae from parasitoid wasps. Curr. Biol. 17, 2105-2116.

Isenberg, K. E. and Meyer, G. E. (1989). Cloning of a putative neuronal nicotinic acetylcholine receptor subunit. J. Neurochem. 52, 988-991.

Ishizuka, T., Kakuda, M., Araki, R., and Yawo, H. (2006). Kinetic evaluation of photosensitivity in genetically engineered neurons expressing green algae light-gated channels. Neurosci. Res. 54, 85-94.

Kalaidzidis, I. V., Kalaidzidis, Y. L., and Kaulen, A. D. (1998). Flash-induced voltage changes in halorhodopsin from Natronobacterium pharaonis. FEBS Lett. 427, 59-63.

Levitan, E. S. and Takimoto, K. (2000). Surface expression of $\mathrm{Kv} 1$ voltage-gated $\mathrm{K}+$ channels is governed by a C-terminal motif. Trends Cardiovasc. Med. 10, 317-320.

Li, X., Gutierrez, D. V., Hanson, M. G., Han, J., Mark, M. D., Chiel, H., Hegemann, P., Land- messer, L. T., and Herlitze, S. (2005). Fast noninvasive activation and inhibition of neural and network activity by vertebrate rhodopsin and green algae channelrhodopsin. Proc. Natl. Acad. Sci. USA 102, 17816-17821.

Li, D., Takimoto, K., and Levitan, E. S. (2000). Surface expression of $\mathrm{Kv} 1$ channels is governed by a C-terminal motif. J. Biol. Chem. 275, 11597-11602.

Ma, D., Zerangue, N., Lin, Y. F., Collins, A., Yu, M., Jan, Y. N., and Jan, L. Y. (2001). Role of ER export signals in controlling surface potassium channel numbers. Science 291, 316-319.

Nagel, G., Brauner, M., Liewald, J. F., Adeishvili, N., Bamberg, E., and Gottschalk, A. (2005). Light activation of channelrhodopsin-2 in excitable cells of Caenorhabditis elegans triggers rapid behavioral responses. Curr. Biol. 15, 2279-2284.

Nagel, G., Szellas, T., Huhn, W., Kateriya, S., Adeishvili, N., Berthold, P., Ollig, D., Hegemann, P., and Bamberg, E. (2003). Channelrhodopsin-2, a directly light-gated cation-selective membrane channel. Proc. Natl. Acad. Sci. USA 100, 13940-13945.

Petreanu, L., Huber, D., Sobczyk, A., and Svoboda, K. (2007). Channelrhodopsin-2-assisted circuit mapping of long-range callosal projections. Nat. Neurosci. 10, 663-668.

Petrecca, K., Miller, D. M., and Shrier, A. (2000). Localization and enhanced current density of the Kv4.2 potassium channel by interaction with the actin-binding protein filamin. J. Neurosci. 20, 8736-8744.

Schroll, C., Riemensperger, T., Bucher, D., Ehmer, J., Voller, T., Erbguth, K., Gerber, B., Hendel, T., Nagel, G., Buchner, E., and Fiala, A. (2006). Light-induced activation of distinct modulatory neurons triggers appetitive or aversive learning in Drosophila larvae. Curr. Biol. 16, 1741-1747.

Stockklausner, C. and Klocker, N. (2003). Surface expression of inward rectifier potassium channels is controlled by selective Golgi export. J. Biol. Chem. 278, 17000-17005.

Stockklausner, C., Ludwig, J., Ruppersberg, J. P., and Klocker, N. (2001). A sequence motif responsible for ER export and surface expression of Kir2.0 inward rectifier $\mathrm{K}^{+}$channels. FEBS Lett. 493, 129-133.

Wang, H., Peca, J., Matsuzaki, M., Matsuzaki, K., Noguchi, J., Qiu, L., Wang, D., Zhang, F., 
Boyden, E., Deisseroth, K., Kasai, H., Hall, W. C., Feng, G., and Augustine, G. J. (2007). High-speed mapping of synaptic connectivity using photostimulation in Channelrhodopsin-2 transgenic mice. Proc. Natl. Acad. Sci. USA 104, 8143-8148.

Weick, J. P., Groth, R. D., Isaksen, A. L., and Mermelstein, P. G. (2003). Interactions with PDZ proteins are required for L-type calcium channels to activate cAMP response elementbinding protein-dependent gene expression. J. Neurosci. 23, 3446-3456.

Zhang, F., Aravanis, A. M., Adamantidis, A., de Lecea, L., and Deisseroth, K. (2007b). Circuitbreakers: Optical technologies for probing neural signals and systems. Nat. Rev. Neurosci. 8, 577-581.

Zhang, Y. and Harris-Warrick, R. M. (2004). An ER export signal accelerates the surface expression of shal potassium channels in pyloric neurons of the lobster stomatogastric ganglion. Pflugers Arch. 447, 401-404.

Zhang, Y. P. and Oertner, T. G. (2007). Optical induction of synaptic plasticity using a lightsensitive channel. Nat. Methods 4, 139-141.

Zhang, F., Prigge, M., Beyriere, F., Tsunoda, S. P., Mattis, J., Yizhar, O., Hegemann, P., and
Deisseroth, K. (2008). Red-shifted optogenetic excitation: A tool for fast neural control derived from Volvox carteri. Nat. Neurosci. 11, 631633.

Zhang, F., Wang, L. P., Boyden, E. S., and Deisseroth, K. (2006). Channelrhodopsin-2 and optical control of excitable cells. Nat. Methods 3, 785-792.

Zhang, F., Wang, L. P., Brauner, M., Liewald, J. F., Kay, K., Watzke, N., Wood, P. G., Bamberg, E., Nagel, G., Gottschalk, A., and Deisseroth, K. (2007a). Multimodal fast optical interrogation of neural circuitry. Nature 446, 633-639.

Zhao, S., Cunha, C., Zhang, F., Liu, Q., Gloss, B., Deisseroth, K., Augustine, G. J., and Feng, G. (2008). Improved expression of halorhodopsin for light-induced silencing of neuronal activity. Brain Cell Biol. 36, In this issue.

Zito, K., Parnas, D., Fetter, R. D., Isacoff, E. Y., and Goodman, C. S. (1999). Watching a synapse grow: Noninvasive confocal imaging of synaptic growth in Drosophila. Neuron 22, 719-729. 